PRAXIS

ecucativa

Universidad Nacional de La Pampa

Facultad de Ciencias Humanas

Instituto de Ciencias de la Educación

para la investigación interdisciplinaria

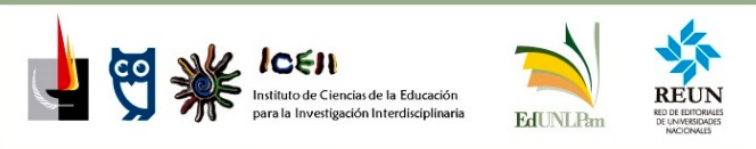

ISSN 2313-934X

SANTA ROSA, LA PAMPA, ARGENTINA

Correo electrónico: iceii@humanas.unlpam.edu.ar

Disponible en https://cerac.unlpam.edu.ar/index.php/praxis

La Formación de docentes en ejercicio a través de la Lesson Study: preocupaciones en una realidad compleja. Artículo de Víctor Miguel Sumba Arévalo. Praxis educativa, Vol. 26, No 1 enero - abril 2022. E - ISSN 2313-934X. pp. 1-17.

\title{
La Formación de docentes en ejercicio a través de la Lesson Study: preocupaciones en una realidad compleja
}

Training of the practicing teacher through the Lesson Study: concerns in a complex reality

Treinamento do professor praticante por meio do Lesson Study: preocupações em uma realidade complexa

\section{Víctor Miguel Sumba Arévalo}

Ministerio de Educación del Ecuador, Ecuador

vimisuare@yahoo.es

ORCID: 0000-0002-8208-5233 


\section{Resumen}

El presente artículo responde a un avance de la investigación doctoral desarrollada en la carrera de Educación Básica, modalidad a distancia, del Plan para la Profesionalización Docente de la Universidad Nacional de Educación-UNAE-Ecuador. Pretende identificar las preocupaciones que presentan los docentes en el contexto de la educación virtual mediante el desarrollo de la metodología Lesson Study, de manera concreta en la fase de problematización. Para tal propósito, desde un enfoque cualitativo y estudio de caso, se analizan dichas preocupaciones manifestadas en entrevistas individuales, grupo de discusión y de los textos escritos. Se pueden observar preocupaciones referidas a las dimensiones del conocimiento práctico: conocimientos, habilidades, emociones, valores y actitudes. Esto lleva a comprender que el rol docente, en la actualidad, se enfrenta a nuevos desafíos y realidades inciertas, concluyendo en la importancia de una formación permanente que involucre las dimensiones mencionadas para indagar y actuar en y sobre necesidades específicas.

Palabras clave: formación de docentes, práctica pedagógica, competencias del docente, eficacia del docente, complejidad.

\section{Abstract}

This article responds to a progress of the doctoral research developed in the Basic Education career, distance modality of the Plan for Teacher Professionalization of the Universidad Nacional de Educación-UNAEEcuador. It aims to identify the concerns presented by teachers in the context of virtual education through the development of the Lesson Study methodology, specifically in the problematization phase. For this purpose, from a qualitative approach and case study, said concerns expressed in individual interviews, group discussion and written texts are analyzed. Concerns can be observed regarding the dimensions of practical knowledge: knowledge, skills, emotions, values and attitudes. This leads to understanding that the teaching role currently faces new challenges and uncertain realities, concluding on the importance of ongoing training that involves the aforementioned dimensions to investigate and act on and on specific needs.

Keywords: teacher training; pedagogical practice; teacher competencies; teacher effectiveness; complexity.

\section{Resumo}

Este artigo responde a um avanço da pesquisa de doutorado desenvolvida na carreira de Educação Básica, modalidade a distância do Plano de Profissionalização Docente da Universidad Nacional de Educación-UNAEEquador. Objetiva identificar as inquietações apresentadas pelos professores no contexto da educação virtual por meio do desenvolvimento da metodologia Lesson Study, especificamente na fase de problematização. Para o efeito, a partir de uma abordagem qualitativa e de um estudo de caso, são analisadas as referidas preocupações expressas em entrevistas individuais, discussão em grupo e textos escritos. Observam-se preocupações quanto às dimensões do conhecimento prático: conhecimentos, habilidades, emoções, valores e atitudes. Isso leva a compreender que a função docente enfrenta atualmente novos desafios e realidades incertas, concluindo sobre a importância de uma formação permanente que envolva as dimensões mencionadas para investigar e atuar sobre necessidades específicas.

Palavras chave: treinamento de professor; prática pedagógica; competências do professor; eficácia do professor; complexidade. 


\section{Introducción}

La presente investigación responde a la necesidad de volver la mirada sobre la formación de docentes en ejercicio desde y para la complejidad. Es así que, como avance de la investigación doctoral titulada: Formación docente: aportes de la Lesson Study en la profesionalización docente. Estudio de caso en la Universidad Nacional de Educación, trata de evidenciar cómo la metodología de investigación acción Lesson Study, en su fase inicial, pone en evidencia las principales preocupaciones del equipo docente ante una nueva realidad compleja: la educación virtual durante la pandemia. Esta formación pretende alejarse de lo tradicional y meramente teórico para llegar a la articulación entre la teoría y práctica. Además, desde nuestro punto de vista, la Lesson Study pretende formar de manera integral considerando otras dimensiones que configuran el rol docente y que permitirían comprender y actuar en la realidad compleja.

El contexto de investigación comprende la carrera de Educación Básica (EB), modalidad a distancia, del Plan para la Profesionalización docente de la Universidad Nacional de Educación UNAE-Ecuador. Esta carrera (junto a la carrera de Educación Intercultural Bilingüe) es ofertada a docentes en ejercicio que no poseen título de tercer nivel, experiencia mínima de cinco años, ejercen sus funciones en el nivel de Educación General Básica (comprende desde el primer al décimo año de educación), entre otras características (UNAE, 2020). La metodología Lesson Study, dentro de la carrera, comprende una asignatura de componente teórico, mientras que el componente práctico se refleja en la asignatura Cátedra Integradora. Por tanto, el seguimiento y registro de la información lo realizamos en este último.

\section{Formación permanente vista desde la complejidad}

Es necesario poner en evidencia nuestro posicionamiento en relación con la formación docente, vista desde el enfoque de la complejidad. Es como hacemos referencia, en primera instancia, a la idea de Gorodokin (2006) al mencionar que "implica una acción profunda ejercida sobre el sujeto, tendiente a la transformación de todo su ser, que apunta simultáneamente sobre el saber-hacer, el saber-obrar y el saber-pensar" (p2). Este punto de partida lleva a tener una mirada integral en la formación docente, alejada de los modelos clásicos como el academicista, tecnicista, artesanal, entre otros, que tienen objetivos específicos: la acumulación del "conocimiento", aplicación de técnicas o recetas, valoración de la práctica ante lo teórico, etc. Lo que se pretende es que la formación influya en cada docente desde un pensamiento crítico para construir nuevos conocimientos con base en la identificación y solución de problemas de la realidad. Esta formación debe ser enfocada desde ámbitos personales y profesionales como el social, cultural, emocional, cognitivo, entre otras que configuran a cada docente. Visto desde la complejidad, es prioritario considerar estos ámbitos, pues un/una docente, en el aula, no omite su bagaje cultural, social, emocional, afectivo, entre otros; más bien, todos estos confluyen en su práctica diaria. Al decir de Álvarez (2018), una formación que influya en el "saber hacer, saber pensar, saber sentir, saber vivir y convivir" (p. 12). 
Es necesario diferenciar entre formación inicial y permanente de los/as docentes. Mientras la primera está orientada a formar nuevos/as docentes desde sus bases teóricas, conceptuales y prácticas, la formación permanente tiene como sujetos de formación a docentes en ejercicio. En este sentido, se concibe a la formación permanente como un espacio para "la problematización de la propia experiencia y del quehacer como fuente para la reconstrucción y apropiación de nuevos saberes, habilidades y actitudes" (Vezub, 2004, p. 6), que completamos con lo que Socket (2012) y Murrel et al. (2010, como se citó en Peña y Pérez Gómez, 2019) denominan disposiciones docentes: al conjunto de actitudes, valores y emociones. Consideramos importantes estas dimensiones, pues condicionan y dan significado al rol docente. En tal sentido, la formación permanente conduce o debería conducir a investigar en el propio contexto y la propia práctica, analizarlo, comprenderlo para reformular la acción docente y enfrentar una realidad cambiante e incierta (Morin, 1990).

Si la propuesta de formación involucra saber identificar los problemas que influyen en la educación, proponer alternativas y solucionarlos, es necesario desarrollar nuevas competencias en el personal docente: la investigativa. Argumentamos la idea del/de la docente investigador/a de Stenhouse (2003) y del/de la profesional reflexivo/a de Schön (1998), pues constituyen la base para problematizar su propia práctica. De los postulados anteriores, y respondiendo al paradigma de la complejidad, se considera que un/a docente investigador/a comprende que el conocimiento educativo no es estático y que este puede ser construido o reconstruido desde la escuela y el equipo docente. Por un lado, la investigación brinda alternativas para enfrentarse a las incertidumbres y realidades, de por sí complejas, del proceso de enseñanza y de aprendizaje. Mientras, la reflexión involucra necesariamente volver la mirada a la práctica desarrollada, pero no desde lo instrumental, sino desde una dimensión conceptual, emocional, ética, actitudinal, etc., como influyentes - conscientes o inconscientes - en la práctica de cada docente.

Por lo mencionado anteriormente, y de acuerdo con Gonfiantini (2016), la formación debe ser vista desde un enfoque que involucre "la reflexión, el diálogo de saberes y las modalidades de aprendizajes que se concreticen en armonía con la esencia de los sujetos, los requerimientos de las sociedades y el necesario respeto a las diferencias" (p. 231-232). En esto radica la importancia de un diálogo entre lo teórico y práctico, entre las diferentes disciplinas encargadas de preparar a las y los docentes en y para la complejidad. La transdisciplinariedad nos Ilevaría a este diálogo necesario.

Otro aspecto importante por considerar en la formación permanente, y este relacionado con la idea del/de la docente investigador/a y reflexivo/a, es su práctica. Este constituye en un espacio y tiempo idóneo para evidenciar los problemas (situaciones) e investigarlos. Es en la práctica donde confluyen y se ponen en evidencia (aunque identificarlas supone una tarea no tan sencilla) las dimensiones culturales, éticas, sociales, epistemológicas, afectivas y cognitivas de cada docente.

Para responder a la realidad actual y compleja desde la formación docente, es necesario romper lo que Pérez Gómez (2020) denomina automatismos cerebrales, que son "imprescindibles 
para actuar con eficacia y economía en la vida cotidiana, pero también al permanecer por debajo de la conciencia son difíciles de detectar y cambiar cuando fuere necesario, cuando se hayan convertido en obsoletos o insuficientes" (p. 9). La realidad educativa, en especial la educación virtual, requiere frenar este automatismo en cuanto al proceso de enseñanza, donde se cuenta con un cúmulo de experiencias que, en su momento -en la presencialidad-, dieron resultados, pero actualmente pueden no tener la misma efectividad, pues la misma realidad requiere de nuevas alternativas de enseñanza para generar aprendizajes en los estudiantes.

Entonces, la formación docente requiere de elementos, procesos, características, etc., para viabilizar el cambio epistemológico que reduce a la enseñanza y aprendizaje como transmisora y receptora de conocimientos, respectivamente. El reto es diseñar experiencias novedosas y exitosas que movilicen a las y los docentes del statu quo y encontrar el sentido de la educación "para ayudar a formar el ciudadano culto, solidario y autónomo que exige la complejidad de este escenario global y digital contemporáneo" (Pérez Gómez, 2020, p. 10). No debemos perder de vista que el fin de la formación docente es perfeccionar sus capacidades, para que, a su vez, generen contextos y experiencias para la construcción de aprendizajes funcionales que aporten significativamente en el proyecto de vida del estudiantado.

Es así que, desde el marco curricular de la UNAE, como parte de la formación docente, se propone la asignatura Lesson Study (incluyen las carreras a distancia) que permite indagar la práctica del equipo docente, reflexionar sobre esta y proponer alternativas de mejora. Esto lleva a indagar sobre el aporte de esta metodología en la formación docente, resaltando la importancia de la participación deliberada de las y los participantes en este proceso.

\section{Lesson Study en la formación de docentes en ejercicio}

Soto y Pérez Gómez (2015) presentan información sobre la procedencia de la Lesson Study, la sitúan en Japón, a finales del siglo XIX, con referentes pedagógicos como la filosofía didáctica de Pestalozzi, aprendizaje basado en la experiencia de Dewey y la investigación acción. Esta metodología requiere la participación directa del personal docente que, tras sus fases, lleva a la reflexión de su propia realidad en el aula. Es así que retomamos la idea presentada en párrafos anteriores en relación al concepto de docente investigador/a de Stenhouse (2003) que, sumada a la investigación acción de Elliot (2015), involucra necesariamente al personal docente en la indagación y construcción del conocimiento, pero una construcción desarrollada desde la participación deliberada, consciente y colaborativa, es decir, "requiere de la asunción de una posición autocrítica y de una disposición para someter el trabajo de cada uno al escrutinio de los demás" (Elliot, 2015, p. 30).

Otra concepción que se relaciona con la Lesson Study es la presentada por Schön (1992), la figura del profesional reflexivo. Este componente - la reflexión-, según Pérez Gómez (2012), es la base para transformar la práctica y, a su vez, constituye un dispositivo de formación. Entonces, investigación y reflexión son componentes esenciales para indagar y transformar el rol docente, pero, ¿cómo se relaciona la investigación acción y la Lesson Study? Pues bien, a esta relación la 
podemos evidenciar en su proceso cíclico, partiendo de la 1) definición del problema; 2) diseño cooperativo de una lección experimental; 3) enseñar y observar en el aula la lección diseñada; 4) recoger las evidencias y discutirlas; 5) analizarla y revisarla; 6) desarrollar la lección revisada en otra clase y observarla; y 7) evaluar y difundir la experiencia. Todas las fases son desarrollas por el equipo docente y desde sus propias necesidades, es así que se emprende en una investigación y formación, dos procesos complementarios entre sí.

En relación con las fases y con el propósito de este artículo, nos centramos en la fase de diagnóstico para conocer y comprender las principales preocupaciones que tienen las y los docentes sobre sus prácticas; pues, como manifestamos anteriormente, en esta realidad compleja y de incertidumbres, surgen nuevas necesidades. Esta fase se convierte en la plataforma para el desarrollo de la investigación acción pues, en ella, emergen las preocupaciones, necesidades, intereses o situaciones que motivan y orientan la acción de docentes. Si bien pueden existir innumerables situaciones, es importante que cada docente focalice el estudio (Vásquez, 2017) para "debatir y formular en grupo los objetivos del aprendizaje a largo plazo de los estudiantes y su relación con la propuesta concreta que queremos trabajar partiendo de nuestras propias prácticas y experiencias" (Soto y Pérez Gómez, 2015, p. 21).

Es a través del diálogo y la reflexión sobre la experiencia, relatos personales escritos u orales (Pérez Gómez et al., 2015) compartidos en grupo, que ayudan a identificar el problema a ser investigado en la Lesson Study. Un aspecto no menos importante en esta fase es el trabajo colaborativo entre docentes. Así, el trabajo entre colegas debe ser deliberado y activo para consolidar las preocupaciones y, posteriormente, las alternativas de acción. Por tanto, esta fase es fundamental para determinar el foco de atención sobre la cual giran las acciones y decisiones posteriores.

Desde el posicionamiento de la complejidad, asumimos que la Lesson Study se aleja de los modelos clásicos de formación, centrados principalmente en el orden teórico, didáctico, curricular, etc. El proceso Lesson Study indaga lo que Soto y Pérez Gómez (2015) denominan las dimensiones del conocimiento y pensamiento práctico: conocimientos, habilidades, valores, actitudes y emociones. Refieren al conocimiento como esquemas, modelos, mapas o guiones que dicen algo de la realidad, es decir, del saber. Por habilidad, destacan a un saber procedimental, sea psicomotor, social o mental, en otras palabras: el saber hacer. En relación con los valores, son principios del ámbito personal y profesional que condicionan la acción docente, mientras que por actitud entienden la disposición de percibir y actuar de manera favorable o desfavorable. Por último, y con el mismo valor e influencia en la práctica docente, están las emociones definidas como las tendencias de aceptación o rechazo de las acciones o decisiones personales o profesionales.

¿Por qué consideramos a la Lesson Study como alternativa para la formación de docentes en ejercicio? Para comprenderlo, traemos a mención la conceptualización del conocimiento práctico: "conjunto de creencias, habilidades, valores, actitudes y emociones que operan de manera automática, implícita, sin necesidad de conciencia y que condicionan nuestra percepción, 
interpretación, toma de decisiones y actuación" (Pérez Gómez et al., 2015, p. 83). Todos estos repertorios de actuación son adquiridos desde la formación inicial y desde la experiencia, todos estos saberes, con el tiempo, se van automatizando en una práctica monótona, sin ser consciente ni actuar ante los cambios constantes de la realidad. En este punto, cobra importancia la investigación acción a través de la Lesson Study, pues su base reflexiva en y sobre la acción (Schön, 1992; Perrenoud, 2007) Ileva a cada docente a darse cuenta de que sus acciones, conocimientos, emociones, actitudes, valores, etc., influyen en su práctica. Este proceso de reflexión conlleva a que el conocimiento y la acción automatizada sean sometidos a discusión, que el/la propio/a docente, y junto a otros/as, cuestionen los resultados evidenciados.

Esta reflexión en y sobre la acción (Schön, 1992) o la teorización de la práctica (Hagger y Hazel, 2006, como se citó en Soto et al., 2021) busca que cada docente reconozca y sustente su práctica con los recursos explícitos e implícitos movilizados por experiencias relevantes en su práctica. Esta teoría debe fundamentar la propia práctica en relación con las acciones desarrolladas en el aula (teorías en uso) y no desde la retórica para adornar la práctica (teorías declaradas). Para enriquecer la experiencia y consolidar la reconstrucción de los conocimientos, habilidades, actitudes, valores y emociones, es necesario que la/el docente experimente la teoría, resultado de la reflexión e investigación, y la convierta en fundamento de la actuación e interpretación (Soto et al., 2021).

Es así que Soto et al. (2015) indican que la Lesson Study lleva a transformar el conocimiento en pensamiento práctico, entendiéndose este último como la combinación del conocimiento práctico más el conocimiento reflexivo sobre la acción; en otras palabras, constituyen los recursos conscientes e inconscientes que influyen en la toma de decisiones. Esto contribuye a indagar, experimentar y detenerse constantemente en la propia práctica para generar contextos y situaciones que propicien el aprendizaje del estudiantado. Por tanto, un aspecto fundamental en la fase inicial de la Lesson Study es identificar categorías relacionadas con las dimensiones del conocimiento práctico que, mediante las acciones desarrolladas en las fases posteriores, ayudarían a configurar y evidenciar el pensamiento práctico.

\section{Metodología}

La presente investigación tiene un enfoque cualitativo que, a través del Estudio de Caso (Flick, 2015; Stake, 2007; Denzin y Lincoln, 2013) en la UNAE, permite focalizar nuestra atención en la carrera de Educación Básica, modalidad a distancia, del Plan para la Profesionalización Docente. El caso está conformado por nueve docentes en ejercicio que, por su condición de estudiantes de la universidad, se encuentran matriculados/as en el octavo ciclo en la asignatura Cátedra integradora: Diseño de propuestas de intervención e investigación educativa, donde se experimenta la teoría presentada en la asignatura Lesson Study. El equipo docente participante cumple sus funciones en la provincia del Cañar-Ecuador, en el nivel Preparatoria y Educación General Básica (ECB). Es necesario mencionar que estos/as conforman tres grupos de tres integrantes cada uno. Estos grupos son constituidos por los/as propios/as docentes mediante la 
orientación de la asignatura Cátedra Integradora. Entre los criterios, constan: trabajar en el mismo nivel (Preparatoria o EGB) o subnivel de ECB (Elemental, Media, Superior) e intereses y preocupaciones comunes.

Como puede comprenderse, el ciclo de formación en la UNAE, el quehacer docente y el proceso de investigación se encuentran directamente influenciados por la modalidad virtual como consecuencia de la emergencia sanitaria. Es en este nuevo contexto donde se indaga ¿cuáles son las preocupaciones que presentan el grupo de docentes en el contexto de la educación virtual mediante el desarrollo de la metodología Lesson Study, de manera concreta en la fase de la problematización? Lo que lleva a identificar las principales preocupaciones que tienen los docentes en la modalidad virtual en la fase de problematización, constituyendo así el objetivo principal para este artículo.

De forma resumida, se menciona que todo proceso de la Lesson Study es desarrollado en modalidad virtual tanto sincrónica como asincrónica. Generalmente, las sesiones asincrónicas están centradas en el diseño y fundamentación teórica de la lección (actividades de la asignatura Cátedra Integradora), mientras que las sincrónicas tratan la socialización del diseño (solicitado por el investigador), desarrollo de la lección, grupos de discusión (análisis de la clase y evaluación de la Lesson Study) y entrevistas individuales. Durante el desarrollo de las fases, se da seguimiento a las dimensiones identificadas inicialmente que constituirán el corpus de la investigación doctoral.

De acuerdo con el objetivo de este texto, se establecen a priori cinco categorías de análisis que responden a las dimensiones del conocimiento y pensamiento práctico y que, de acuerdo con Vásquez et al. (2014), el rol docente sería la manifestación de estas. Por tanto, indagamos las preocupaciones docentes expresadas en relación con los conocimientos, habilidades, valores, actitudes y emociones. Para el proceso de análisis, lo presentamos en tal orden, pero comprendemos que todas estas forman un entretejido de factores personales y profesionales y convergen entre ellas en el rol docente. Esta indagación se desarrolla específicamente en la fase de diagnóstico que realizan los docentes como punto de partida de la Lesson Study, mediante grupo de discusión (GD-LS), entrevista semiestructurada (Entrev1) y análisis de documento (Act.1.6.C-I), este último corresponde a la actividad evaluativa presentada por el grupo en la asignatura Cátedra Integradora.

Para la identificación de grupos, se utiliza la codificación G1LS, G2LS y G3LS mientras que, para el personal docente, se utiliza D1-G1LS, donde D1 identifica al/a la docente y G1LS el grupo al que pertenece. Para el registro y análisis de datos, se cuenta con la autorización respectiva de cada docente, dirección de la carrera y coordinación de investigación de la UNAE.

\section{Resultados}

De acuerdo con las categorías anteriormente señalas, se pueden evidenciar varias preocupaciones en esta nueva realidad de las y los docentes participantes. En primer lugar, en relación con la dimensión de conocimientos, manifiestan sentirse preocupados/das en el avance de los aprendizajes en la modalidad virtual (D2, D3, D4, D5, D6, D7, D8, D9. Entrev1, 2020), esto 
debido a la inasistencia a los encuentros sincrónicos. También autoidentifican una limitación en su práctica docente en cuanto al conocimiento y manejo de la tecnología: "ahora más que nunca necesitamos estar capacitados porque los niños manejan muchísimo la tecnología, entonces si es importante en el aprendizaje" (D3-G1LS, Entrev1, 2020); "no todos estamos en esa capacidad de manejar lo que es la tecnología, estamos muy lejos de manejar la tecnología" (D9-G3LS, 2020). De forma concreta, los tres grupos LS manifiestan el desconocimiento y uso de las plataformas virtuales para el trabajo sincrónico (GD-LS1, 2 y 3, 2020). Todo lo anterior constituye una limitante al momento de desarrollar el proceso de enseñanza y de aprendizaje, sobre todo en esta situación compleja e impredecible, muy alejados de la presencialidad y del aula.

Otro factor que se identifica es en cuanto al manejo de la tecnología por parte del estudiantado o representantes. Es así que, desde la percepción del equipo docente participante, manifiestan que;

Las plataformas hoy en día son algo importante para trabajar, pero hay mucho desconocimiento hablando sobre los estudiantes y padres de familia. Muchos de ellos no saben cómo entrar a las plataformas y no solo eso, son de recursos económicos bajos, no tienen acceso a internet. (D6-G2LS, Entrev1, 2020)

En el mismo sentido, pero en otras palabras la, D8 (G3LS, Entrev1, 2020) manifiesta "para mis estudiantes, creo que es algo nuevo para ellos, porque ellos no saben manejar la plataforma virtual, igual para los padres de familia, es algo dificultoso". Es evidente esta preocupación en relación con la tecnología, tanto por docentes, representantes y estudiantes. Conviene mencionar que las y los docentes valoran el medio tecnológico como una posibilidad de generar aprendizajes mínimos, encuentran en ello una oportunidad de continuidad educativa: "gracias a la tecnología estamos tratando de llegar a ellos, porque si no hubiera estos recursos entonces ahí mucho más se agravaría la situación" (D1-G1LS, Entrev1, 2020); "la tecnología también es una parte fundamental para poder llegar a los estudiantes" (D3-G1LS, Entrev1, 2020). En el mismo sentido, la D3 (G1LS, Entrev1, 2020) manifiesta, en relación a la tecnología, "es un medio que en estas circunstancias para la interacción de alumnos-maestro, pero en sí, en sí, no es tan fructífera como estar directamente con el estudiante". Además, identificamos ciertas creencias que configuran su acción: "no es tan importante cómo aprende el estudiante, sino cómo enseña el docente" (GDG1LS, 2020) y comprenden que "las expectativas del docente influyen en el rendimiento académico de los estudiantes" (GD-G2LS, 2020).

En relación con las habilidades, podemos relacionar con la preocupación presentada anteriormente, pues manifiestan una limitación en el uso de la tecnología donde, indudablemente, se ve condicionado el saber y saber hacer, un ejemplo de ello es lo manifestado por la D5:

Yo, más que nada, me siento un poco frustrada porque realmente, para nosotros, poder desarrollar unas clases virtuales, tenemos que estar diestros en el dominio de las plataformas virtuales, pero lamentablemente esto es una debilidad por parte del 
Ministerio de Educación por no solventarnos en nuestras capacitaciones de medios virtuales. (G2LS, Entrev1, 2020)

Muestran preocupación respecto a su rol, pues la modalidad afecta al proceso de enseñanza y de aprendizaje desarrollado en el aula "que, mediante la educación virtual o a distancia, se agranda el problema debido a que no existe una guía presencial del docente y no se logran aprendizajes significativos" (GD-G1LS, 2020). Emergen nuevas necesidades en el equipo docente del G2LS que se centran en desarrollar la Lesson Study en la asignatura de Matemática y, con ello, evidencian dificultades en el proceso de enseñanza y aprendizaje al resolver problemas, razonamiento y dificultad en desarrollar las fases de la Matemática. Adicional a ello, demuestran interés en su rol de guía, y comprenden que su apoyo en esta nueva realidad es fundamental al mencionar que "el aprendizaje de un estudiante se ve impactado por el apoyo que recibe del docente" (GD-G2LS, 2020).

Mientras que, en la dimensión de las emociones, en la educación virtual, se muestran preocupaciones afectivas, en la relación docente y estudiante, manifiestan el limitado contacto físico, kinestésico y auditivo (CD-G1LS, 2020). Otra docente manifiesta: "estoy contenta porque sigo trabajando con mis estudiantes, pero no al $100 \%$ porque no es lo mismo estar detrás de una computadora que estar directamente con mis estudiantes en el aula" (D2-G1LS, Entrev1, 2020); "para mí, lo más importante es trabajar con el niño en forma presencial, de estar pendiente de ellos, de motivarles, de estar acompañándoles, de estar sintiendo lo que es el afecto" (D3-G1LS, Entrev1, 2020); y "estresante, estresante porque, porque no puedo comunicarme con mis 25 estudiantes que yo tengo, de los cuales solamente once o doce máximo que actúan, que conectan" (D9-G3LS, Entrev1, 2020). Por otro lado, ciertas/os docentes experimentan emociones vinculadas a la falta de atención por parte del estudiantado en los encuentros sincrónicos (GDG2LS, 2020) y, de manera concreta, resaltamos lo expresado por la D1: "no hay esa debida atención como es trabajar en el aula, es bien diferente, es bien diferente, no hay cómo llegar a ellos [...], sin embargo, hay que animarnos, reanimarnos y buscar la manera de seguir adelante" (G1LS, Entrev1, 2020).

En la dimensión de valores, se puede identificar, en el G1LS y G3LS, la importancia que otorgan a la familia durante la modalidad virtual como guías en el aprendizaje de los estudiantes. En palabras de algunas/os docentes participantes: "es un rol muy importante, el apoyo de ellos es la base para el aprendizaje del niño, de la niña. Es un rol primordial que ahora nos tocó vivir y hablo por mí también que soy madre de familia" (D1-G1LS, Entrev1, 2020); "El rol del padre de familia pasó a ser el rol del docente, nosotros ahorita estamos siendo los guías porque ellos pasaron a ser docentes porque están haciendo la mayoría de trabajo" (D2-G1LS, Entrev1, 2020); "el rol de la familia es un trabajo fundamental en casa, es que si no hay el apoyo de la familia [...] es muy difícil que avance" (D3-G1LS, Entrev1, 2020); y "Ahora sí digo que el apoyo incondicional, un apoyo gigante [...]. Si ahorita existe descuido de una madre, de un padre, entonces el niño no va a tener un buen aprendizaje" (D9-G3LS, Entrev1, 2020). 
Lo que se puede observar en la dimensión de las actitudes es la dependencia con las percepciones de cada grupo que, en primera instancia, lo relacionamos como teoría proclamada. Así, para el G1LS (CD, 2020), su actitud se relaciona con la importancia que otorgan al aprendizaje de la Matemática; pues, la consideran "esencial para la vida". Al presentar este interés en particular por la asignatura y considerarla esencial, podría influir en sus formas de abordar la asignatura y en las acciones a desarrollar para garantizar el aprendizaje de los estudiantes en esta modalidad. Para el G2LS, el interés radica en proponer actividades para que cada estudiante sea el/la protagonista de su propio aprendizaje en la modalidad virtual (CD, 2020). Al tener la idea consolidada sobre el/la estudiante como sujeto constructor de su propio aprendizaje, llevaría a generar propuesta de experiencias relevantes para el estudiantado. Ya en el G3LS, podemos evidenciar la actitud en cuanto a su rol: "Un gran maestro hace del aprendizaje algo divertido, estimulador e interesante y que lleva al estudiante al éxito académico y hace del aula un lugar acogedor, también influye la motivación, la organización y la relación personal" (GD, 2020).

El objetivo de la primera fase de la Lesson Study, como se mencionó anteriormente, es definir el problema. De la discusión y trabajo colaborativo de docentes y como resultado de cada uno de los grupos, se refleja en identificar el foco de atención que conducirá las demás fases de la Lesson Study. Así, el G1LS (Act.1.6.C-I, 2020) desarrolla, en la asignatura de Matemática, en relación con el aprendizaje y enseñanza de la suma sin reagrupación con números hasta el 19 (con decena) en el segundo de básica. El G2LS (Act.1.6.C-I, 2020) centra su atención en la enseñanza y aprendizaje de resolución de problemas matemáticos con números hasta el nueve (con unidades) en segundo de básica. Por último, el G3LS centra su atención en desarrollar el razonamiento lógico matemático con estudiantes del primer año de educación básica en el ámbito Lógico Matemático (Act.1.6.C-I, 2020).

Todas las preocupaciones, necesidades e intereses presentadas por el equipo docente emergen de un nuevo contexto, una nueva realidad para el desarrollo del proceso de enseñanza y de aprendizaje. Las dimensiones del conocimiento práctico, expresadas e identificadas, nos llevan a comprender la complejidad de la profesión docente y no desde una mirada simplificadora. Pero ahora, qué comprendemos por estos resultados. A continuación, presentamos una discusión que gira en torno a estos.

\section{Discusión}

Del proceso de investigación en la fase inicial de la Lesson Study desarrollada con docentes en formación de la carrera de EB del Plan para la Profesionalización Docente de la UNAE y de acuerdo con el objetivo, se han identificado las preocupaciones en la modalidad virtual relacionadas con las dimensiones del conocimiento y pensamiento práctico. Estas dimensiones apuntan a considerar aspectos personales y profesionales del personal docente como influyentes en su práctica; en otras palabras, este dispositivo de formación señala a una formación más amplia y no al reduccionismo de lo académico o técnico. 
En primera instancia, referido a los conocimientos, podemos comprender la preocupación de docentes en el manejo de las plataformas y recursos digitales, pues la nueva modalidad se desarrolla a través de este medio. Esta necesidad ha flotado en esta realidad, pone en evidencia la limitada formación y habilidad en el manejo de los recursos tecnológicos; esto también lo relacionamos a la escasa preocupación (longitudinal) desde el sistema de educación en ofertar programas de formación más amplios y consolidados en este tema. Desde el inicio de la pandemia hasta la presente fecha, el Ministerio de Educación del Ecuador (Mineduc) propone una variedad de cursos para fomentar el uso de las plataformas y recursos tecnológicos (Mineduc, s.f.), pero, como puede verse, aún existe esta necesidad en las/os docentes. Relacionado a esto, y como parte de las preocupaciones del equipo docente, está el escaso conocimiento y uso de estos recursos por parte del estudiantado y representantes; pues, la nueva realidad requiere necesariamente de este recurso. Esto lo relacionamos a la escasa o nula formación que reciben los y las estudiantes sobre el uso de la tecnología en la escuela: años atrás, se eliminó la asignatura Informática de la malla curricular (generalmente en instituciones de EGB fiscales). Todo lo anterior, y durante las primeras semanas o meses de la modalidad virtual, lleva a que docentes, estudiantes y padres y madres de familia desarrollen la enseñanza y el aprendizaje mediante experimentaciones, ensayos y errores.

Se resalta la creencia del G1, poco argumentada en relación con dar mayor importancia a la forma de enseñar sobre la forma de aprender. Si bien la Lesson Study contribuye en la práctica docente, no deja de lado la comprensión sobre cómo las/os estudiantes participan, construyen o consolidan sus aprendizajes (Soto et al., 2015), esta creencia del cuerpo docente puede constituir una condicionante en el proceso de enseñanza y aprendizaje que se desarrolla en la educación virtual, lo cual constituye un foco de atención durante el proceso. Ya investigaciones como las desarrolladas por Rosenthal y Jacobson (1968), Mares et al. (2009), García et al. (2014), entre otros, consideran fundamental las expectativas del/de la profesor/a en relación a sus estudiantes; pueden apoyar o limitar el adecuado aprendizaje.

Relacionado con la dimensión anterior y ligado con las habilidades, los y las docentes manifiestan dificultades en el manejo de las plataformas para garantizar el aprendizaje de los estudiantes, pues "las nuevas condiciones han requerido que el profesorado utilice plataformas y metodologías virtuales con las que no necesariamente se encontraba familiarizado" (CEPAL, 2020, p. 11). Otro factor que consideran importante en su rol docente es el contacto presencial con el estudiantado para garantizar los aprendizajes. Lo anterior visibiliza, por parte del equipo docente, poca seguridad en los recursos tecnológicos para garantizar aprendizajes, requieren de una mediación presencial para desarrollarlos, ejemplo: las fases de la matemática. Esta creencia puede relacionarse con el desconocimiento en el uso de los recursos tecnológicos, lo que lleva a considerar la presencialidad como única opción para la enseñanza y aprendizaje.

Mientras que las emociones del equipo docente se centran en la no relación directa con sus estudiantes, muestran preocupaciones y ciertas angustias. Podemos identificar que la afectividad de docentes a estudiantes juega un papel fundamental en el desarrollo de las 
actividades. Lo que convendría pensar, en esta realidad - aún incierta-, sobre cómo garantizar o poner en evidencia las relaciones afectivas entre docentes y estudiantes. En el contexto de la pandemia, la UNICEF enfatiza que "Enseñar y aprender son procesos complejos que involucran vínculos de reciprocidad, deseos y búsquedas [...] resulta central cuidar el bienestar emocional de quienes están participando de la experiencia pedagógica" (2020, p. 4). Todo esto pone en evidencia la afectación emocional del personal docente y, paradójicamente, es quien contribuye en acciones para garantizar el bienestar emocional de las familias ante la incertidumbre de esta realidad. Volviendo la mirada a la relación docente y estudiante, la CEPAL manifiesta que:

Desde el punto de vista pedagógico, la virtualidad supone el riesgo de pérdida del vínculo presencial y puede generar tensiones por la sobreexposición de docentes y estudiantes, o por las dificultades para mantener la relación y la mediación pedagógicas. Esto es especialmente cierto en los niveles iniciales de educación, en particular en el preescolar y el primario, en los que se requiere un trabajo coordinado con padres, madres o cuidadores para el acompañamiento y la mediación de los procesos de niños y niñas. (2020, p. 10)

En relación con los valores, los y las docentes resaltan la importancia de la familia en el aprendizaje de cada estudiante, atribuyen a la familia el rol de educadores mientras que el/la docente cumple con el rol de guía. No es ajeno que la fase de "Aprendemos juntos en casa", en Ecuador, involucra directamente a la familia como contexto y promotores del desarrollo de las niñeces. Docentes en general, y participantes de esta investigación en particular, promueven actividades asincrónicas y sincrónicas para guiar el proceso de aprendizaje y, en ciertas ocasiones, el personal docente guía a los padres y madres de familia sobre cómo llegar al aprendizaje. Para resaltar la percepción que tienen algunos/as docentes participantes de la familia, traemos a mención: "Si ahorita existe descuido de una madre, de un padre, entonces el niño no va tener un buen aprendizaje" (D9-G3LS, Entrev1, 2020); "a veces, necesitan bastante, bastante el apoyo de los papás, estén ahí por lo menos los papás escuchando la clase para luego ellos poderles ayudar en las tareas o en las actividades que uno se les deja" (D7-G3LS, Entrev1, 2020); "El rol de ellos es dedicarse a los estudiantes y ser, como se dice, un hilo conductor entre el docente y el estudiante" (D5-G2LS, Entrev1, 2020); y "es un rol muy importante, el apoyo de ellos es la base para el aprendizaje del niño, de la niña" (D1-G1LS, Entrev1, 2020). Otro punto necesario por resaltar es que las y los docentes relacionan su profesión con su rol en la familia, hablan desde su experiencia en el hogar, esto argumenta que la acción docente está influenciada desde sus ámbitos personales y juega un papel importante en sus decisiones.

Ciertas percepciones que tiene el equipo docente sobre el estudiantado o las asignaturas se transforman en actitudes hacia su función docente y las/os predispone para actuar de una manera determinada. Al otorgar importancia a la matemática como "esencial para la vida" (G1LS), aspirar a que el estudiante sea el "propio constructor" de su aprendizaje (G2LS) y la idea de "un gran maestro" es quien propone actividades estimulantes para el aprendizaje, influyen, desde nuestro entender, en sus actividades y decisiones favoreciendo el desarrollo del aprendizaje. Estas 
actitudes, de una u otra manera, se convierten en un hilo conductor del accionar docente. Para llegar a ese ideal, los docentes transformarían su práctica y, evidentemente, es uno de los objetivos de la Lesson Study.

Resaltamos la interrelación entre las dimensiones del conocimiento práctico, no se dan de forma independiente, las habilidades requieren de los conocimientos. A su vez, estas se ven influenciadas por las emociones, actitudes y valores, y no necesariamente en este orden. En fin, lo que resaltamos es el entretejido de estas dimensiones, desconocer esto sería como argumentar a favor de una formación centrada solo en lo académico, técnico, emocional de forma independiente y no desde la recursividad como propone Morin (1990). Entonces, la Lesson Study deja en evidencia la convergencia de los conocimientos, habilidades, actitudes, emociones y valores que dan sentido a la práctica docente y que son el reflejo de estas dimensiones. Ya en la investigación presentada por Vásquez se muestra esta convergencia:

Entendemos que en la realidad se presentan en continuidad y en interacción permanente; componentes que además se manifiestan en la vida cotidiana en gradaciones distintas y fluctúan desde los procesos más netamente cognitivos y abstractos hasta los fenómenos más nítidamente emocionales. (2017, p. 270)

Por último, referimos a la definición del problema en cada uno de los grupos como objetivo de esta fase. Por un lado, el trabajo colaborativo y la empatía ayuda a generar la discusión y el compartir de experiencias exitosas o no. Es dialogando, discutiendo, argumentando, reflexionando sobre sus prácticas en el aula y el aprendizaje de los estudiantes que el cuerpo docente prioriza los problemas que consideran urgentes que, en el caso del G1LS, podemos relacionarlo con la actitud hacia la Matemática al considerarla esencial para la vida, dejando en evidencia su influencia en las decisiones del equipo docente. Por otra parte, los y las docentes son conscientes de las exigencias para la educación virtual en cuanto a conocimientos y habilidades tecnológicas y, en relación con ello, los grupos LS autoidentifican cierta limitación. Consideramos importante esto ya que son conscientes de que la realidad ha cambiado y requieren de otras competencias para viabilizar el proceso de enseñanza y de aprendizaje.

\section{Conclusiones}

Podemos manifestar la diversidad de preocupaciones que presentan los y las docentes en una realidad compleja e incierta en la modalidad virtual. Las dimensiones del conocimiento práctico enfocadas desde la Lesson Study nos permiten identificar varios aspectos personales y profesionales que influyen y confluyen en la tarea del/de la docente. En nuestro caso, las nuevas - preocupaciones emergen del contexto de la modalidad virtual y, en ella, inquietudes referidas a los conocimientos teóricos y procedimentales en cuanto a los recursos tecnológicos; actitudinales por la propia percepción a la asignatura -importancia de la matemática- y sobre el rol del/de la docente para generar aprendizajes significativos; valores al reconocer la importancia de la familia para garantizar el aprendizaje del estudiantado; y emocionales por el no contacto directo con cada estudiante. Identificar estas preocupaciones nos proporciona información sobre 
los aspectos que deben ser considerados en la formación docente, en nuestro caso, la formación de docentes en ejercicio para fortalecer, configurar o reconfigurar su rol, entendida esta como la expresión del conocimiento y pensamiento práctico.

Este avance de investigación deja a la luz grandes desafíos en la realidad educativa en general y la formación permanente en particular. Pues, no bastará en propuestas de formación desde lo teórico (academicista), metodológico (técnico) o práctico (artesanal) para responder a las necesidades del equipo docente y de la educación. La Lesson Study contribuye a viabilizar una formación en y para la práctica desde las necesidades e intereses reales de los y las docentes, donde permite articular lo teórico y práctico, dimensiones personales y profesionales, aula y contexto, entre otras.

Esta primera fase ayuda a indagar el punto de partida para el recorrido de la formación e investigación acción. Con esto, argumentamos a favor de pensar y repensar en una formación e investigación articulada. Este es el inicio de una indagación y, durante el desarrollo de las fases de la Lesson Study, permitirá bucear a mayor profundidad en las acciones, pensamientos, actitudes, valores y emociones de docentes y comprender cómo afrontan esta realidad y cómo esta, mediante la reflexión, contribuye a modificar la práctica para transformar el conocimiento en pensamiento práctico.

La fase de diagnóstico comprende un espacio fundamental en el desarrollo de la Lesson Study que, a través del diálogo, discusión y reflexión entre colegas, se explicitan las preocupaciones, intereses, necesidades, problemas, etc., que identifica cada docente en su práctica. De esta manera, se considera a este como primer momento de teorización de la práctica (en nuestro caso, a través de GD-LS, Entrev1 y Act.1.6.C-I) de la cual se focaliza la situación a resolver, no desde miradas externas ni de problemas evidenciados por otros, sino desde los/as propios/as docentes. Esto difiere de los programas de formación o capacitaciones tradicionales que, si bien responden a problemas generales, no necesariamente responden a problemas particulares que los y las docentes evidencian en el aula. Así, el compartir y discutir experiencias entre docentes del grupo contribuyen en la identificación del problema y comprender el entretejido de factores que influyen en ello. 


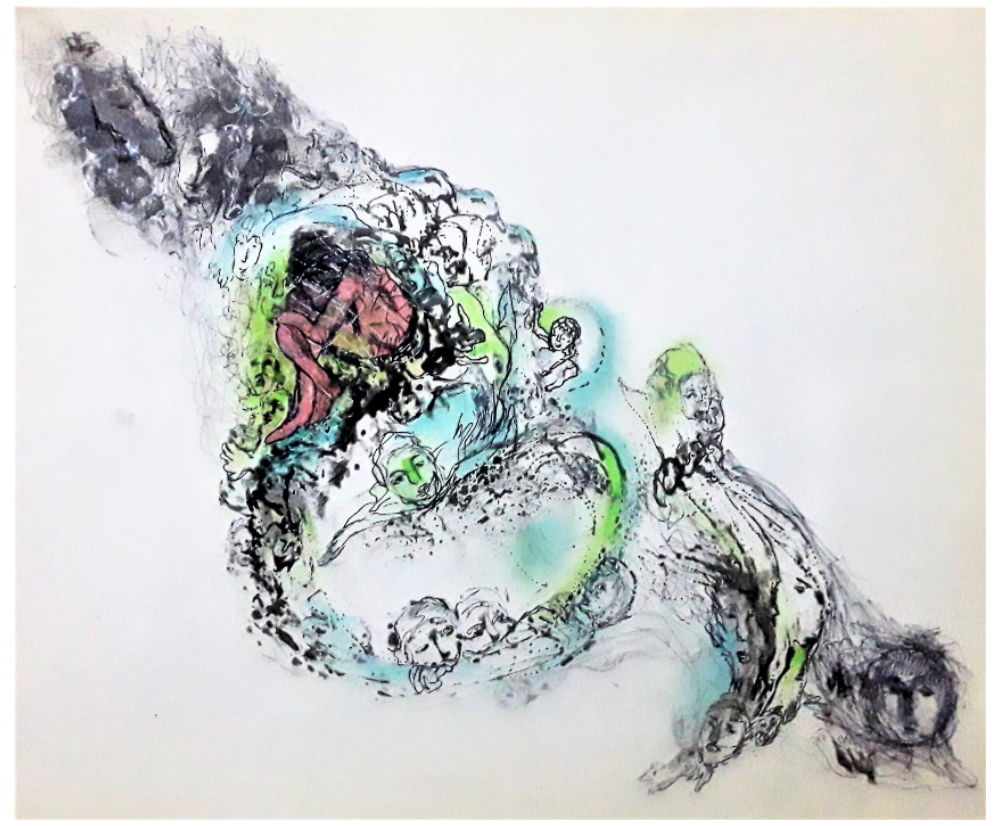

Agobio, técnica mixta. Adriana Chavarri

\section{Bibliografía}

Álvarez, F. (2018). Transformar la educación para transformar la sociedad. UNAE-EP.

https://unae.edu.ec/wpcontent/uploads/2020/03/TrasnformarLaEducacionParaTransformarlaSociedad.pdf

CEPAL. (2020). La educación en tiempo de la pandemia de COVID-19. https://repositorio.cepal.org/bitstream/handle/11362/45904/1/s2000510_es.pdf Denzin, N. y Lincoln, Y. (2013). Las estrategias de investigación cualitativa. Gedisa.

Elliott, J. (2015). Lesson y learning Study y la idea del docente como investigador. Revista Interuniversitaria de Formación del Profesorado, 29(3), 29-46. https://www.redalyc.org/articulo.oa?id=27443871003 Flick, U. (2015). El diseño de investigación cualitativa. Morata.

García, E., García, A., y Reyes, J. (2014). Relación maestro alumno y sus implicaciones en el aprendizaje. Ra Ximhai, 10(5), 279-290. https://www.redalyc.org/articulo.oa?id=46132134019

Confiantini, V. (2016). Formación docente y diálogo de saberes en el kairos educativo. Sophia, Colección de Filosofía de la Educación, (21), 229-245. https://www.redalyc.org/articulo.oa?id=441849209010 Gorodokin, I. (2006). La formación docente y su relación con la epistemología. Revista Iberoamericana de Educación, 375), 1-9. https://rieoei.org/historico/deloslectores/1164Gorodokin.pdf

Mares, A., Martínez, R. y Rojo, H. (2009). Concepto y expectativas del docente respecto de sus alumnos considerados con necesidades educativas especiales. Revista mexicana de investigación educativa, 14(42), 969-996. http://www.scielo.org.mx/scielo.php?script=sci_arttext\&pid=S140566662009000300016\&lng=es\&tIng=es.

Ministerio de Educación del Ecuador. (s.f.). Plataforma mecapacito.

https://mecapacito.educacion.gob.ec/nuestros-cursos/

Morin, E. (1990). Introducción al pensamiento complejo. Gedisa. 
Peña, N. y Pérez Gómez, Á. (2019). Las disposiciones subjetivas de los docentes en la superación de las resistencias al cambio ante procesos cíclicos de formación basados en la investigación (Lesson Study): Estudio de un caso. Revista Complutense de Educación, 30(2), 569-587.

http://dx.doi.org/10.5209/RCED.57780

Pérez Gómez, Á. (2012). Educarse en la era digital. Morata.

Pérez Gómez, Á. (2020). Los desafíos educativos en tiempos de pandemias: ayudar a construir la compleja subjetividad compartida de los seres humanos. Praxis educativa, 24(3), 1-24.

https://doi.org/10.19137/praxiseducativa-2020-240302

Pérez Gómez, Á., Soto, E. y Serván, M. (2015). Lesson Studies: re-pensar y re-crear el conocimiento práctico en cooperación. Revista Interuniversitaria de Formación del Profesorado, 29(3), 81-101.

https://www.redalyc.org/articulo.oa?id=27443871006

Perrenoud, P. (2007). Desarrollar la práctica reflexiva en el oficio de enseñar. Graó.

Rosenthal, R y Jacobson, L. (1968). Pygmalion in the classroom: teacher expectation and pupils' intellectual development. Holt, Rinebart and Winston.

Schön, D. (1992). La formación de profesionales reflexivos. Paidós.

Soto, E. y Pérez Gómez, Á. (2015). Lessons Studies: un viaje de ida y vuelta recreando el aprendizaje comprensivo. Revista Interuniversitaria de Formación del Profesorado, 29(3), 15-28.

https://www.redalyc.org/articulo.oa?id=27443871002

Soto. E., Maldonado, G., Márquez, A. y Peña, N. (2021). Reconstruyendo el conocimiento práctico en confinamiento. Una experiencia de enseñanza en la formación inicial de docentes. RED. Revista de Educación a Distancia, 65(21), 1-39. https://doi.org/10.6018/red.450621

Stake, R. (2007). Investigación con estudio de casos. Morata.

Stenhouse, L. (2003). Investigación y desarrollo del currículo. Morata.

UNICEF. (2020). Contención emocional de equipos directivos y herramientas para docentes y familia: el acompañamiento a la comunidad educativa en un contexto de emergencia.

https://www.unicef.org/argentina/sites/unicef.org.argentina/files/2020-06/EDU-equipos-conduccionCovid-3.pdf

Universidad Nacional de Educación. (2020). Carrera de Profesionalización. UNAE. Recuperado el 7 de abril de 2020 de https://unae.edu.ec/oferta/educacion-basica-profesionalizacion/?portfolioCats=144

Vásquez, K., Peña, N., Rodríguez, J., Becerra, A., García, S. y Pérez Gómez, Á. (2014). Conocimiento Práctico y rol docente. Experiencias de reconstrucción a través de un proceso de formación colaborativa basada en Lesson Study. Siete estudios de casos. XIII Congreso Internacional de Formación del Profesorado. Santander. Colombia.

https://riuma.uma.es/xmlui/bitstream/handle/10630/8686/M4\%20SES5115.pdf?sequence=1\&isAllowed=y Vásquez. K. (2017). La reconstrucción del conocimiento práctico en docentes de infantil con relación a la metodología de enseñanza a través de la Lesson Study. Dos estudios de caso [Tesis de doctorado]. Universidad de Málaga https://riuma.uma.es/xmlui/bitstream/handle/10630/15968/TD_VASQUEZ_SUAREZ_Kena.pdf?sequence=3 Vezub, L. (2004). Las trayectorias de desarrollo profesional docente: algunos conceptos para su abordaje. Revista del Instituto de Investigaciones en Ciencias de la Educación, 22, 3-12. http://repositorio.filo.uba.ar:8080/xmlui/handle/filodigital/9899 